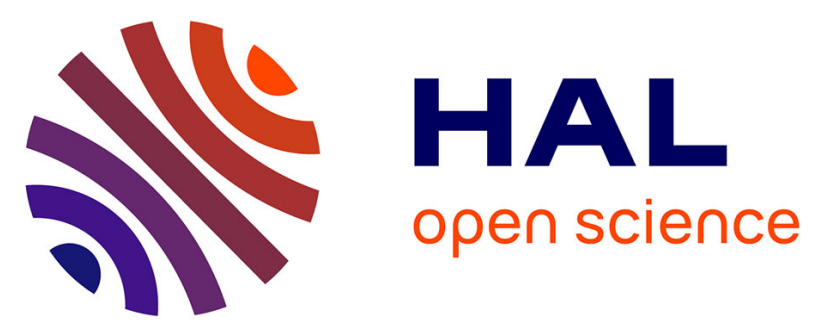

\title{
Polarization-dependent vacuum-ultraviolet reflectometry using elliptically polarized synchrotron radiation
} Alexander Gottwald, Françoise Bridou, Mireille Cuniot-Ponsard, Jean-Michel Desvignes, Simone Kroth, Udo Kroth, Wolfgang Paustian, Mathias Richter, Hendrik Schöppe, Thornagel Reiner

\section{To cite this version:}

Alexander Gottwald, Françoise Bridou, Mireille Cuniot-Ponsard, Jean-Michel Desvignes, Simone Kroth, et al.. Polarization-dependent vacuum-ultraviolet reflectometry using elliptically polarized synchrotron radiation. Applied optics, 2007, 46 (32), pp.7797-7804. hal-00575921

\section{HAL Id: hal-00575921 \\ https://hal-iogs.archives-ouvertes.fr/hal-00575921}

Submitted on 8 Mar 2012

HAL is a multi-disciplinary open access archive for the deposit and dissemination of scientific research documents, whether they are published or not. The documents may come from teaching and research institutions in France or abroad, or from public or private research centers.
L'archive ouverte pluridisciplinaire HAL, est destinée au dépôt et à la diffusion de documents scientifiques de niveau recherche, publiés ou non, émanant des établissements d'enseignement et de recherche français ou étrangers, des laboratoires publics ou privés. 


\title{
Polarization-dependent vacuum-ultraviolet reflectometry using elliptically polarized synchrotron radiation
}

\author{
Alexander Gottwald, ${ }^{1, \star}$ Françoise Bridou, ${ }^{2}$ Mireille Cuniot-Ponsard, ${ }^{2}$ Jean-Michel Desvignes, ${ }^{2}$ \\ Simone Kroth, ${ }^{1}$ Udo Kroth, ${ }^{1}$ Wolfgang Paustian, ${ }^{1}$ Mathias Richter, ${ }^{1}$ Hendrik Schöppe, ${ }^{1}$ \\ and Reiner Thornagel ${ }^{1}$ \\ ${ }^{1}$ Physikalisch-Technische Bundesanstalt (PTB), Abbestraße 2-12, 10587 Berlin, Germany \\ 2Laboratoire Charles Fabry de l'Institut d'Optique, UMR 8501 du CNRS, de l'Institut d'Optique et de l'Université Paris Sud, \\ RD 128, 91127 Palaiseau Cedex, France \\ *Corresponding author: alexander.gottwald@ptb.de
}

Received 22 June 2007; revised 19 September 2007; accepted 24 September 2007; posted 25 September 2007 (Doc. ID 84394); published 1 November 2007

\begin{abstract}
In the laboratory of the Physikalisch-Technische Bundesanstalt (PTB) at the Berlin electron-storage ring BESSY II, a procedure has been developed to investigate the dependence of vacuum-ultraviolet reflection on polarization. It is based on characterizing the elliptically polarized synchrotron radiation at PTB's normalincidence monochromator beamline for reflectometry by means of polarization-sensitive photodetectors. For this purpose, the polarization dependency in the detector responsivity was determined at a small, low, solid angle of acceptance for the synchrotron radiation, i.e., within the orbital plane of the storage ring where the degree of linear polarization is known to be almost 100\%. Our method allows the polarization dependence of reflection samples to be measured with relative standard uncertainties ranging from $2.4 \%$ to $11 \%$ in the spectral range between 60 and $160 \mathrm{~nm}$. The method has been applied to the optimization of polarizing mirrors at the Lyman- $\alpha$ wavelength of $121.6 \mathrm{~nm}$. (C) 2007 Optical Society of America
\end{abstract}

OCIS codes: $120.3940,120.5700,120.5410,260.7210,340.6720$.

\section{Introduction}

The characterization of optical materials, i.e., the measurement of reflectance or transmittance and the determination of optical constants, in the vacuumultraviolet (VUV) and extreme-ultraviolet (EUV) spectral regions at wavelengths below $200 \mathrm{~nm}$ has attracted growing interest during the past decade. Industrial applications, e.g., photolithography, refer to the excimer wavelengths (157, 172, and $193 \mathrm{~nm})$ and, moreover, to $13 \mathrm{~nm}$. In research fields such as extraterrestrial solar astronomy, this wavelength region is of growing importance, too, as demonstrated by several instruments recently put into operation [1]. With decreasing wavelength, the practical problems of accurate measurements become an increasing challenge, mainly due to the air absorption and the nonavailability of stable broadband or tunable ra-

0003-6935/07/327797-08\$15.00/0

(C) 2007 Optical Society of America diation sources. Using monochromatized synchrotron radiation from the Berlin electron storage ring (BESSY) II and high-vacuum reflectometers, Physikalisch-Technische Bundesanstalt (PTB) has established the characterization of materials, filters, and mirrors from the ultraviolet to the x-ray spectral range. For the wavelength interval between 40 and $400 \mathrm{~nm}$, a normal-incidence monochromator beamline delivers spectrally dispersed radiation for this purpose (Fig. 1) [2]. Synchrotron radiation as emitted from storage rings is linearly polarized in the storage ring plane, while the out-of-plane radiation shows elliptical polarization. In the case of the beamline regarded here, an off-axis aperture defines the vertical acceptance angle from 0.2 to $3.0 \mathrm{mrad}$ with respect to the storage ring plane. This off-axis aperture is needed to suppress the high portion of on-axis x-ray radiation of the BESSY II storage ring, which would induce an intolerable heat load to the first mirror. The polarization characteristics behind the off-plane aperture can be calculated using Schwinger's theory 


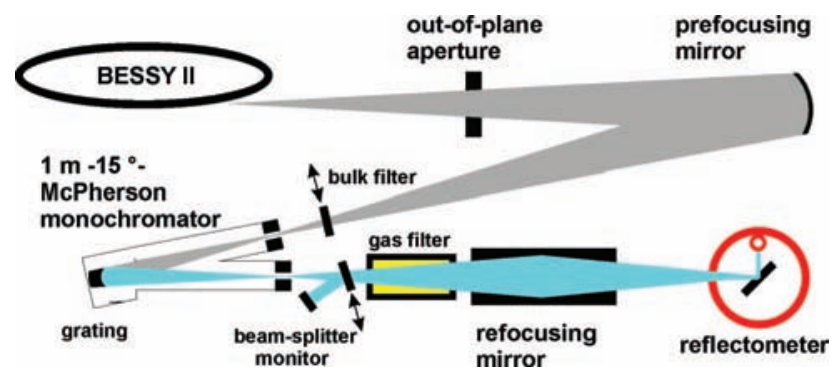

Fig. 1. (Color online) Scheme of the VUV reflectometry beamline at the PTB laboratory at BESSY II.

[3]. However, it is adulterated by the gratings (three different ones are in use, which differ in density and coating), the filter and the beam-splitter plate characteristics, and by the gracing-incidence refocusing mirror, resulting in elliptical polarization to a wavelength-dependent degree at the beamline focus. The resulting polarization cannot be easily calculated, particularly due to the grating, leading to the implication that the beamline up to now has not been suitable for use in the direct determination of any polarization-dependent optical characteristics. These characteristics have come more and more into the focus of interest: One prominent example is the investigation of the optical anisotropy in $\mathrm{CaF}_{2}$ at $157 \mathrm{~nm}$ wavelength [4,5] with its severe impact on the development of commercial photolithography at that wavelength.

Investigations of the polarization characteristics of VUV and soft $x$-ray synchrotron radiation (polarimetry), from the beginnings in the 1960s [6,7], were carried out by several groups in the context of the development of suitable polarizers with different instrumentations, such as multiple reflectors $[8,9]$ or multilayer devices $[10,11]$, mostly restricted to a limited wavelength interval. Here, we present a method to determine the polarization of the radiation used for reflectance determination in our apparatus, which consequently enables us to distinguish $s$ and $p$ polarization in VUV reflectometry between 40 and $400 \mathrm{~nm}$ with a defined uncertainty budget. The method is not based on the knowledge of any optical constants of a polarizing material, but uses the basic properties of synchrotron radiation as given by Schwinger's theory. An example of an application to solar physics investigation at $121.6 \mathrm{~nm}$ is given.

\section{Experimental Method}

For the determination of sample reflectances and transmittances at the VUV beamline in the PTB laboratory at BESSY II [12], a VUV reflectometer system is in use [13]. The reflectometer with its degrees of freedom is shown schematically in Fig. 2. For the reflectance determination, the incident beam intensity and the reflected beam intensity are measured with the same semiconductor photodiode. The measured signals are normalized to monitor signals in order to compensate for variations in the synchrotron radiation intensity, i.e., the monotonic decrease due
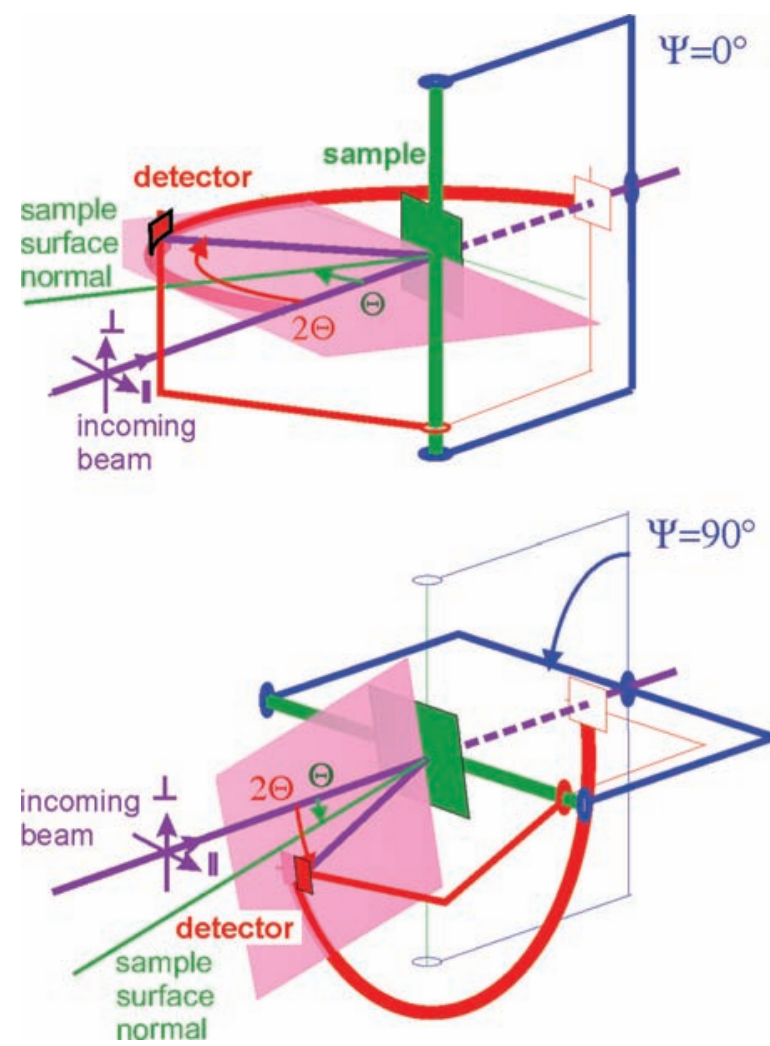

Fig. 2. (Color online) Schematic view of the $s$ - and $p$-polarization ( $\Psi=90^{\circ}$ and $0^{\circ}$, respectively) measurement geometries using the PTB VUV reflectometer.

to the limited lifetime of the stored electron beam in the storage ring. Depending on the wavelength region, either the ring current itself, a photodiode current from a beam-splitter monitor, or the photoemission current of the refocusing mirror are used for normalization. To measure the reflected beam intensity, the photodetector arm is pivoted to the angle of $2 \Theta$ with respect to the incoming beam axis, while the sample is under the reflectance angle of $\Theta$ to fulfill the reflection condition. $\Theta$ can be chosen from approximately $2^{\circ}$ to $85^{\circ}$. In the near-normal incidence case, the angle is limited due to the shadowing at small angles of the direct beam by the detector. For reflectance angles smaller than $10^{\circ}$, the difference between $s$ and $p$ reflectance can commonly be neglected, and the angle $\Psi$ is set to zero ( $p$ geometry for assumed linear polarization in the storage ring plane). The detector is adjusted with its surface normal almost parallel to the beam axis, therefore polarization effects in the detector efficiency are not taken into account. The effect of a $5^{\circ}$ tilt that is implemented to avoid backreflections from the detector surface to the sample-which would adulterate the measurement-is negligible. The measured photocurrent of the detector, then, is proportional to the radiant power. Polarization-dependent measurements (distinguishing between $s$ and $p$-polarization in the sample reflectance) can be performed by rotating the reflectometer around the beam axis by $\Psi=90^{\circ}$, thus switching from $p$ to $s$ geometry. It presupposes linear polarized 
radiation, but in the real case of elliptical polarization, for known ellipticity, the polarization variances may be taken into account and corrected, as described below.

\section{A. Formalism}

For the determination of the polarization-dependent reflectivity of a sample the degree of polarization of the monochromatized synchrotron radiation must, in particular, be taken into account. For a radiation detector, and for the sample under investigation as well, the polarization components will be distinguished, here, by the electrical field vector perpendicular to the incident plane (indexed "s") or parallel to it (indexed " $p$ "). In general, the monochromatized synchrotron radiation shows an elliptical polarization, so that the electrical field vector of the propagating radiation wave can be split into two linear components along the main axes of the polarization ellipse. In the unaltered case, i.e., without optical components that change the beam polarization, the polarization ellipse main axis will be parallel to the storage ring plane (horizontal, indexed "|"), and perpendicular to it (vertical, indexed “ $\perp$ "). As we will see later, this may be slightly changed by the beamline; nevertheless, we will keep the terminology for easier understanding.

The polarization in the reflectance of a mirror can be described by the polarization parameter $P_{R}$, which is defined by the reflectances $R_{s}$ and $R_{p}$ in $s$ and $p$ geometry, respectively,

$$
P_{R}:=\frac{R_{s}-R_{p}}{R_{s}+R_{p}} .
$$

It is correlated with the total reflectivity $R$ by

$$
\begin{aligned}
& R_{s}=\left(1+P_{R}\right) R, \\
& R_{p}=\left(1-P_{R}\right) R,
\end{aligned}
$$

so that

$$
R=\left(R_{s}+R_{p}\right) / 2 .
$$

The responsivity polarization of the photodetector, $P_{D}$, and the polarization degree $P_{\Phi}$ of the monochromatized radiation are defined analogously:

$$
\begin{aligned}
P_{D} & :=\frac{s_{s}-s_{p}}{s_{s}+s_{p}}, \\
P_{\Phi} & :=\frac{\Phi_{\|}-\Phi_{\perp}}{\Phi_{\|}+\Phi_{\perp}} .
\end{aligned}
$$

$\Phi_{\|, \perp}$ denotes the radiant power with the respective polarization of the electric field vector of the monochromatized synchrotron radiation. $s_{s}$ and $s_{p}$ are the respective detector responsivities for the $s$ - and $p$-polarization geometry for reflectance of the detector surface. The radiation reflected by the sample has a resulting polarization $P_{\Phi, R}$, which is a combination of the polarization of the incident radiation and the polarization in reflection:

$$
P_{\Phi, R}=P_{\Phi} P_{R}=\frac{R_{s} \Phi_{\|}+R_{p} \Phi_{\perp}-R_{p} \Phi_{\|}-R_{s} \Phi_{\perp}}{R_{s} \Phi_{\|}+R_{p} \Phi_{\perp}+R_{p} \Phi_{\|}+R_{s} \Phi_{\perp}} .
$$

Regarding the (standard) geometry for $\Psi=0^{\circ}$, the plane of incidence on the sample surface is parallel to the storage ring plane. Therefore, the $s$ reflectance $R_{s}$ is only relevant to the $\Phi_{\perp}$ component, while $R_{p}$ describes the reflectivity for the $\Phi_{\|}$component, so that the reflected intensity is given by

$$
\Phi_{R, \Psi=0^{\circ}}=R_{s} \Phi_{\perp}+R_{p} \Phi_{\|} .
$$

For $\Psi=90^{\circ}$, it is vice versa, since turning the reflectometer setup by $90^{\circ}$ exchanges the $s$ and $p$ geometry:

$$
\Phi_{R, \Psi=90^{\circ}}=R_{p} \Phi_{\perp}+R_{s} \Phi_{\|} .
$$

By inserting Eqs. (6) and (7) into Eq. (5), one obtains

$$
P_{\Phi, R}=\frac{\Phi_{R, \Psi=90^{\circ}}-\Phi_{R, \Psi=0^{\circ}}}{\Phi_{R, \Psi=90^{\circ}}+\Phi_{R, \Psi=0^{\circ}}}
$$

$P_{\Phi, R}$ therefore is determined by a measurement of the reflected intensities for $\Psi=0^{\circ}$ and $90^{\circ}$. However, to determine $P_{R}=P_{\Phi, R} / P_{\Phi}$ [see Eq. (5)], an additional independent measurement of $P_{\Phi}$ is needed.

\section{B. Polarization of the Detector Responsivity}

In our method, $P_{\Phi}$ is determined with the aid of the responsivity polarization $P_{D}$ of photodiodes, measured at a different beamline with well-defined, known polarization. The PTB beamline for VUV source calibration at BESSY II [2] is carefully aligned to the plane of the storage ring with a small vertical acceptance below $1 \mathrm{mrad}$, so that only $\Phi_{\|}$is present in the beam, and therefore $P_{\Phi}=1$ can be assumed (an uncertainty for this will be given in Section 3). For a

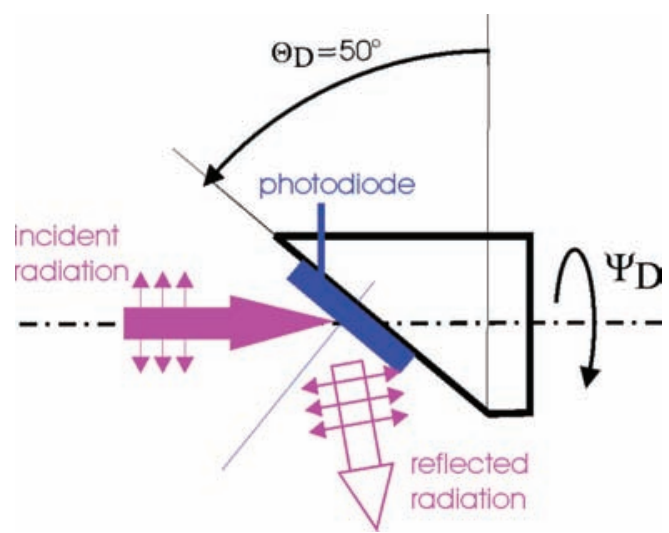

Fig. 3. (Color online) Geometry of the tilted photodiode used for the polarization measurements. 
photodiode with the detector surface tilted by a sufficiently large, fixed angle $\Theta_{D}$ (see Fig. 3), the polarization dependence in responsivity accounts, so that the measured detector photocurrent $I^{D}$ shows a polarization $P_{I}^{D}$ :

$$
P_{I}^{D}=P_{\Phi} P_{D}=\frac{s_{s} \Phi_{\|}+s_{p} \Phi_{\perp}-s_{p} \Phi_{\|}-s_{s} \Phi_{\perp}}{s_{s} \Phi_{\|}+s_{p} \Phi_{\perp}+s_{p} \Phi_{\|}+s_{s} \Phi_{\perp}} .
$$

The polarization dependence is mainly induced by the polarization-dependent reflection properties of the diode's surface. Analog to Eq. (8), $P_{I}^{D}$, in the general case, is therefore determined by measurements with rotating the diode around the beam axis by the angle $\Psi_{D}$ (but leaving $\Theta_{D}$ unchanged). In the case of the vanishing $\Phi_{\perp}$ component $\left(P_{\Phi}=1\right), P_{D}$ is directly given by $P_{I}^{D}$ :

$$
P_{I, \Phi_{\perp}=0} D=\frac{s_{s} \Phi_{\|}-s_{p} \Phi_{\|}}{s_{s} \Phi_{\|}+s_{p} \Phi_{\|}}=\frac{s_{s}-s_{p}}{s_{s}+s_{p}}=P_{D}
$$

Figure 4 shows the measured result for a particular photodiode used in this setup. For the measurements reported here, a silicon $p-n$ photodiode from Ioffe
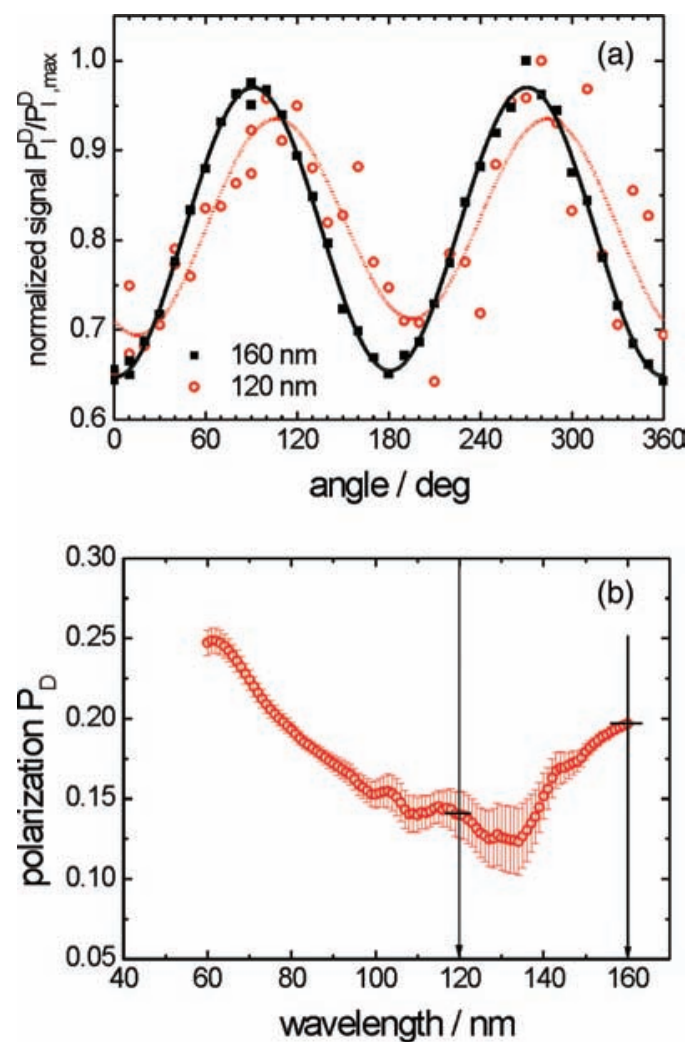

Fig. 4. (Color online) (a) Photodiode signal (normalized to ring current and maximum) with linearly polarized radiation at wavelengths of 120 (open symbols) and $160 \mathrm{~nm}$ (closed symbols) versus rotation angle $\Psi_{D}$. The lines are the results of a cosine function fit to the data points. (b) Responsivity polarization of the photodiode with $50^{\circ}$ tilted mounting versus wavelength. The arrows mark the wavelengths of 120 and $160 \mathrm{~nm}$ where the measurements in (a) were taken.
Physico-Technical Institute was used [14]. This type of detector was selected since it showed the highest polarization sensitivity in combination with reasonable radiation hardness in the wavelength region under investigation. In Fig. 4(a), the photodiode current is shown normalized to its relative maximum versus the angle $\Psi_{D}$ at two different wavelengths (120 and $160 \mathrm{~nm}$ ). The lines shown are results of a cosine function fit. From the fit, the responsivity polarization $P_{D}$ is determined for the particular wavelength. The positions of the minima and maxima and the respective phase shift of the curves indicate the direction of the polarization ellipse main axes. From the geometry it is expected that the minima are at $0^{\circ}$ and $180^{\circ}$, corresponding to the supposed linear polarization of the synchrotron radiation in the horizontal (storage ring) plane. The optical elements in the beamline may rotate the polarization axis, leading to a phase shift of the cosine function. At $120 \mathrm{~nm}$, the experimental data show large variances, corresponding to a large uncertainty of the fitted parameter for the polarization. The variances are due to the only small responsivity of the photodiode around $120 \mathrm{~nm}$, resulting in a high noise in measurement. Figure 4(b) shows the wavelength dependence of the detector polarization, i.e., the results for $P_{D}$ taken at different wavelengths in the interval between 60 and $160 \mathrm{~nm} . P_{D}$ varies between 0.1 and 0.25 in these wavelength regions, with the minimum at $\sim 130 \mathrm{~nm}$.

C. Polarization of the Monochromatized Radiation in the Vacuum-Ultraviolet Reflectometry Beamline

Using the same detector under the same defined tilt angle $\Theta_{D}$ as in Subsection 2.B, the polarization $P_{\Phi}$ of the monochromatized synchrotron radiation in the VUV reflectometry beamline ("beamline polarization") could then be determined. Equation (9), with the now known value of $P_{D}$, was now resolved concerning $P_{\Phi}$ again by measuring the dependence of the detector photocurrent $I^{D}$ on the rotation angle $\Psi_{D}$ of the tilted detector around the beam axis. Figure 5(a) shows the result of this measurement. The resulting polarization in the normalized photodiode current is here named $P_{I}^{\Phi}$, to be distinguished by the superscript from $P_{I}^{D}$. Equation (9) remains valid in this case, too. Comparing Fig. 5(a) to the curves shown in Fig. 4(a), it can be seen that the relative change between minima and the normalized maximum is much smaller; this is a direct consequence of $P_{\Phi} \neq 1$, i.e., the existence of polarization components perpendicular to the horizontal plane. Moreover, the polarization ellipse is obviously rotated so that the main axes are at $\Psi=-10^{\circ}$ and $80^{\circ}$ rather than in exact horizontal and vertical directions $\left(0^{\circ}\right.$ and $\left.90^{\circ}\right)$. Again, this rotation may be due to the grating and filter optical properties that change the polarization characteristics of the beam. Nevertheless, we will continue the use of the $\|$ and $\perp$ notation; however, in the polarization measurements the angles $\Psi=0^{\circ}$ and $90^{\circ}$ will be replaced by $-10^{\circ}$, and $80^{\circ}$, so that the argumentation leading to Eq. (8) remains valid. 

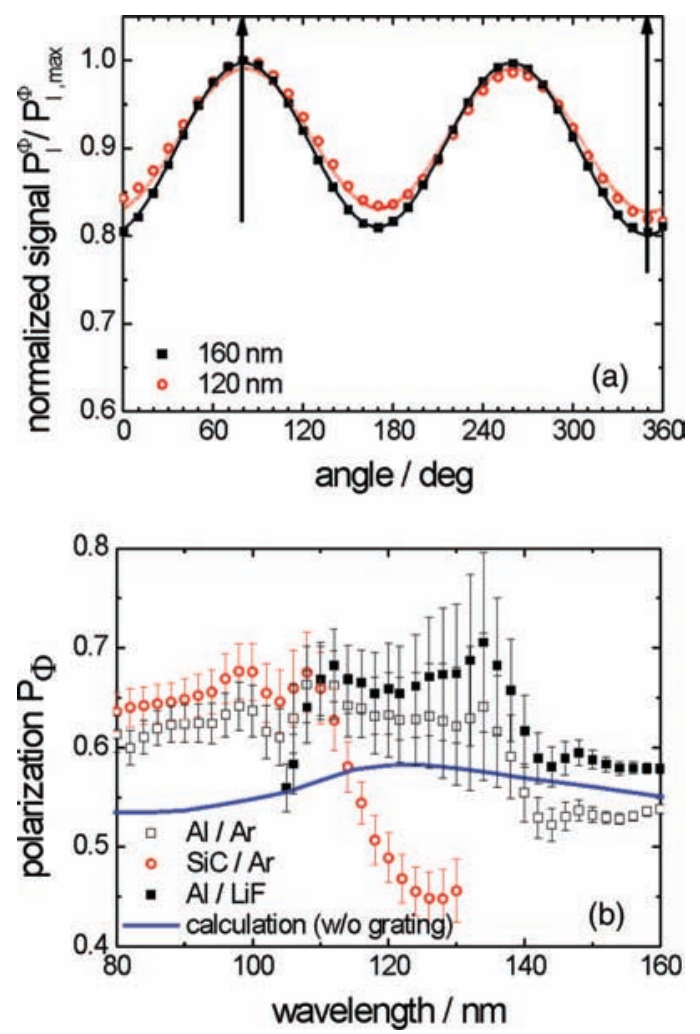

Fig. 5. (Color online) (a) Photodiode signal (normalized to ring current and maximum) with elliptically polarized radiation of the VUV reflectometry beamline at wavelengths of 120 (open symbols) and $160 \mathrm{~nm}$ (closed symbols) versus rotation angle $\Psi_{D}$. The lines are the results of a cosine function fit to the data points. The arrows indicate the resulting measurement positions for $s$ - and $p$-polarization $\left(80^{\circ}\right.$ and $\left.-10^{\circ}\right)$. (b) Polarization of the VUV reflectometry beamline for different optical configurations in the 80 to $160 \mathrm{~nm}$ wavelength regime. The optical surfaces (i.e., premirror, grating) are coated either with aluminum (Al, squares) or silicon carbide (SiC, circles), and for spectral purity filtering either an argon gas (Ar, open symbols) or a lithium fluoride filter ( $\mathrm{LiF}$, closed symbols) is used. The line is a calculation based on Schwinger's theory (see text).

Figure 5(b) presents the resulting polarization $P_{\Phi}$ in the 80 to $160 \mathrm{~nm}$ wavelength range, measured for three different optical configurations of the beamline. Shown are the values for a 600 grooves $/ \mathrm{mm}$ aluminum-coated grating in combination with either an Ar-absorption filter (Al/Ar) or $\mathrm{LiF}$ bulk filter $(\mathrm{Al} / \mathrm{LiF})$, both used for the suppression of higherorder radiation from the monochromator. Moreover, the values for a 1200 grooves $/ \mathrm{mm} \mathrm{SiC-coated} \mathrm{grating}$ in combination with the Ar gas filter are shown. Obviously, the grating as well as the filtering alters the beamline polarization. Additionally, Fig. 5(b) shows a calculated curve for the polarization of the radiation. The emission of synchrotron radiation from dipole magnets can be calculated using Schwinger's theory when the storage ring parameters and optical configurations (apertures) are known. This calculation does not take into account any alteration by the grating, however the reflection at the refocusing gracingincidence mirror in the beamline is considered here by calculation of the polarization-dependent reflectance using tabulated optical constants incorporated in the REFLEC code [15]. For most wavelengths and optical configurations in the regime under investigation, the measured polarization of the monochromatized radiation is higher than that resulting from the calculation of dipole emission. Remembering that pure linear (horizontal) polarization corresponds to $P_{\Phi}=1$, the higher polarization values indicate a suppression of the vertical polarization component by the beamline optics.

\section{Aspects of the Uncertainty Budget}

This section deals with the uncertainty budget for the determination of the reflectance polarization. As shown in Section 2, the determination of the polarization dependence in reflectance $P_{R}$ is experimentally complex, with several independent parameters to be determined. In principle, three measurements are needed, which contribute as sources of uncertainty: (i) the uncertainty in the determination of $P_{\Phi, R}$ by reflectance measurements with $\Psi=-10^{\circ}$ and $80^{\circ}$ [Eq. (8)], (ii) the uncertainty in the polarization dependence of the signal $P_{I}^{\Phi}$ for the determination of the polarization of the monochromatized radiation $P_{\Phi}$, and (iii) the uncertainty in the measurement of the detector responsivity polarization $P_{I}^{D}$. The latter two uncertainties [(ii) and (iii)] are derived from the cosine function fits to the measured data shown in Figs. 4(a) and 5(a), respectively. For (i), only the two largest uncertainty contributions arising from the measurement of the reflected intensity are of significant relevance; these are the uncertainty in the diode signal current measurement, and in the normalization to the monitor signal from the incident radiant power. The first includes the contribution arising from higher-order diffracted radiation and false light in the monochromatized synchrotron radiation beam at the VUV beamline, which depend, in particular, on the wavelength range, i.e., the optical configuration (mirror, grating, filters). Using different filter systems, the beamline is optimized for spectral purity. Between 115 and $160 \mathrm{~nm}$, high-order radiation is suppressed using a $\mathrm{MgF}_{2}$ filter, while at lower wavelengths a gas absorption cell filled with $\mathrm{Ar}$ is used. The uncertainty budget for the determination of the (total) reflectance is described in detail elsewhere [16].

The main contributions to the uncertainty in the determination of $P_{R}$ are summarized in Table 1 , as an example of measurements at 121.6 and $160 \mathrm{~nm}$ wavelength. Additionally, the deviation from pure linear polarization at the VUV source calibration beamline used for the determination of the detector response polarization is taken into account with $0.6 \%$, resulting from calculations with the given geometry using the Schwinger equation. It is obvious that this polarization deviation, as well as the current measurements and the normalization to the incoming beam power for the determination of $P_{\Phi, R}$ according to Eq. (8), only have minor contributions to the total uncer- 
Table 1. Main Contributions to the Uncertainty Budget in the Determination of Reflectance Polarization at the Wavelengths of 121.6 and $160 \mathrm{~nm}$

\begin{tabular}{|c|c|c|}
\hline \multirow[b]{2}{*}{ Quantity } & \multicolumn{2}{|c|}{$\begin{array}{c}\text { Relative Uncertainty } \\
\text { Contribution } \\
(\%)\end{array}$} \\
\hline & $121.6 \mathrm{~nm}$ & $160 \mathrm{~nm}$ \\
\hline Diode signal current measurement & 0.3 & 0.3 \\
\hline $\begin{array}{l}\text { Stability of normalization to beam } \\
\text { power }\end{array}$ & 0.4 & 0.4 \\
\hline $\begin{array}{l}\text { Signal polarization } P_{I}^{D} \text { from } \\
\quad \text { detector }\end{array}$ & 10.8 & 2.3 \\
\hline $\begin{array}{l}\text { Signal polarization } P_{I}^{\Phi} \text { from } \\
\quad \text { radiation }\end{array}$ & 2.2 & 0.4 \\
\hline Beam polarization & 0.6 & 0.5 \\
\hline Reflectance polarization & 11.0 & 2.4 \\
\hline
\end{tabular}

tainty. The main contribution arises from the determination of the responsivity polarization $P_{D}$ of the photodiode [Fig. 4(a)]. Around $120 \mathrm{~nm}$, where the polarization in responsivity as well as the absolute responsivity value are quite low, the relative standard uncertainty in the determination of $P_{D}$ amounts up to $\sim 11 \%$. In contrast, the measurement of the polarization in the photodiode current $P_{I}^{\Phi}[$ Fig. 5(a)] at the VUV reflectometry beamline has a much lower uncertainty of $2.3 \%$ at the same wavelength. This is due to the higher photon flux available at that beamline and wavelength, resulting in a smaller noise effect in the measured detector current. The given uncertainties result from a least-squares-fit procedure of the cosine function to the measurement points. Between 120 and $140 \mathrm{~nm}$, the total relative uncertainty in reflectance polarization determination is not below $10 \%$, while at other wavelengths much lower uncertainties are possible, e.g., $2.4 \%$ in total at $160 \mathrm{~nm}$. In the critical region, the uncertainty reached with our current method therefore can be regarded quite high, compared to recent experiments in this wavelength region using laboratory discharge sources and/or dedicated polarizers for polarimetry $[17,18]$. So, the use of the tilted photodiode for polarization detection is quite effective in realization; however, it is hampered by the relatively large uncertainties in the critical wavelength region around $120 \mathrm{~nm}$. In contrast to methods using dedicated polarizers, our method has the advantage of determining the polarization in reflectance without including any optical constants; thereby the resulting uncertainty does not depend on the uncertainty in their determination.

\section{Example}

The polarization characteristics in VUV reflectance are of particular interest for narrowband reflectors with thin-layer overcoats or multilayer mirrors. Through the design of the layers, not only the center wavelength may be selected, but also the polarization characteristics can be formed [19]. Moreover, the polarization measurements provide additional informa- tion on the multilayer structure that may be useful to optimize coating processes. Therefore, we will demonstrate here the measurement on a thin-layer sample prepared by the Institut d'Optique Théorique et Appliquée (IOTA).

\section{A. Making an Analyzer at $\lambda=121.6 \mathrm{~nm}$}

In the VUV domain, the hydrogen Lyman-alpha $(\mathrm{H}$ $\mathrm{Ly}-\alpha$ ) radiation at a wavelength of $\lambda=121.6 \mathrm{~nm}$ is of particular interest for solar physics. The direction of polarization of this solar radiation is sensitive to the intensity of the ambient magnetic field. The analysis of the rotation in polarization is a way to study the magnetic field in the solar corona. In the example given here, the goal is to make an analyzer setup at $121.6 \mathrm{~nm}$, formed by one or two successive mirrors. A compromise has to be found in order to optimize the $R_{s} / R_{p}$ ratio and the reflectance (better than 0.25) under an incidence angle, required to be lower than $70^{\circ}$.

At this wavelength, the refractive indices of most materials are complex. However, the total extinction of $p$ polarization cannot be achieved by reflection on a bulk material due to the fact that the Fresnel coefficient cannot be zeroed. This total extinction can be obtained by depositing a fluoride thin film of appropriate thickness. The determination of this latter thickness necessitates a precise knowledge of the fluoride indices at $121.6 \mathrm{~nm}$ [20]. The resulting polarization-dependent reflectivity can be improved by stacking several fluoride thin films. In the exampleé shown here, only a single layer of $\mathrm{MgF}_{2}$ has been deposited on a $\mathrm{SiO}_{2}$ substrate.

\section{B. Experimental Procedure}

The films of $\mathrm{MgF}_{2}$ are evaporated on glass or $\mathrm{SiO}_{2}$ substrates, $2 \mathrm{~cm}$ in diameter, at room temperature in an ultrahigh vacuum chamber equipped with a quartz thickness monitor controlled electron gun. The background pressure in the chamber is lower than $10^{-6} \mathrm{~Pa}$. Using grazing $\mathrm{x}$-ray reflectometry at $\lambda=0.154 \mathrm{~nm}$, we determined the thickness, interfacial roughness, and the complex index of refraction (at the source wavelength) of the films by fitting a

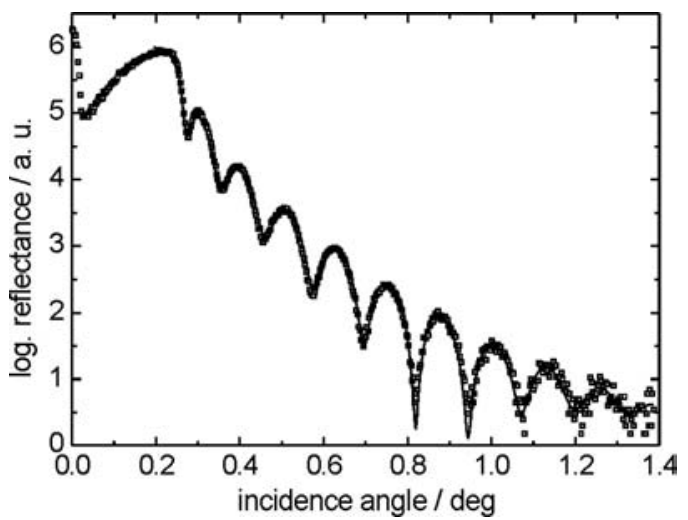

Fig. 6. Experimental (symbols) and fitted (continuous curve) grazing reflectance versus incidence angle at $\lambda=0.154 \mathrm{~nm}$ (x-ray reflectometry) of a $\mathrm{MgF}_{2} / \mathrm{SiO}_{2}$ sample. 
Table 2. Fit Parameters for the $\mathrm{MgF}_{2} / \mathrm{SiO}_{2}$ Sample Properties From X-ray Reflectometry Data at $0.154 \mathrm{~nm}$ (Fig. 6 ) and VUV Reflectometry Data at $121.6 \mathrm{~nm}$ (Fig. 7) ${ }^{a}$

\begin{tabular}{cccc}
\hline $\begin{array}{c}\text { Layer and Wavelength } \\
(\mathrm{nm})\end{array}$ & $\begin{array}{c}\text { Thickness } \\
(\mathrm{nm})\end{array}$ & $\begin{array}{c}\text { Roughness } \\
(\mathrm{nm})\end{array}$ & $\begin{array}{c}\text { Refractive Index } \delta \\
\left(10^{-6}\right)\end{array}$ \\
\hline $\begin{array}{c}\text { Substrate } \\
0.154 \mathrm{~nm}\end{array}$ & $\infty$ & 0.3 & 6.49 \\
$121.6 \mathrm{~nm}$ & $\infty$ & 0.4 & 2.18 \\
$\mathrm{MgF}_{2}$ & & & 0.30 \\
$0.154 \mathrm{~nm}$ & 33.8 & 1.4 & 8.80 \\
$121.6 \mathrm{~nm}$ & 33.6 & 1.0 & 0.16 \\
0
\end{tabular}

${ }^{a} \delta$ and $\beta$ are, respectively, the real and imaginary parts of the complex index $n=1-\delta-\mathrm{i} \beta$.

calculated reflectivity curve to the experimental one [21]. An example of experimental and fitted grazing reflectivity is shown in Fig. 6 for a $\mathrm{MgF}_{2}$ film on $\mathrm{SiO}_{2}$ substrate. The parameter values that yield the best fit are displayed in Table 2. The mirror is designed for an optimized reflectance at $\mathrm{H} \mathrm{Ly}-\alpha$. It was investigated regarding the angular dependence of reflectance at incidence angles between $\Theta=5^{\circ}$ and $85^{\circ}$.

The at-wavelength measurement at $\mathrm{H}$ Ly- $\alpha$ was performed according to the procedure described in the experimental section (Section 2), i.e., $P_{\phi, R}$ was determined by two measurements with $\Psi \stackrel{\phi, R}{=}-10^{\circ}$ and $+80^{\circ}$ [see Eq. (8)]. From Eq. (5) it can be seen that $P_{R}$, and therefore the reflectances $R_{s}$ and $R_{p}$, can be determined by dividing $P_{\Phi, R}$ by $P_{\Phi}$ (see Fig. 5) with respect to the optical configuration of the beamline.

\section{Results}

The result of the $\mathrm{H} \mathrm{Ly}-\alpha$ reflectance measurement is depicted in Fig. 7. The sample shows a quite typical reflectance behavior, with a local minimum of the $p$ reflection around $\Theta=66^{\circ}$, and a monotone increase in $s$ reflectance. The reflectance polarization shows a maximum of $(0.88 \pm 0.09)$ at $67^{\circ}$ reflectance angle. The (total) absolute reflectance [Eq. (3)] is almost constant up to $\Theta=50^{\circ}$ and amounts to $(0.200$ \pm 0.003 ) for this sample; at higher angles it rises up to $(0.48 \pm 0.01)$ at $\Theta=82^{\circ}$. The thickness and the complex index of refraction determined by fitting a

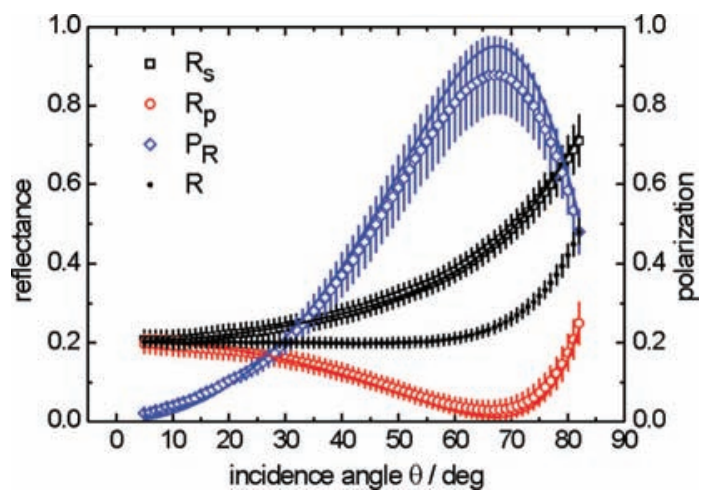

Fig. 7. (Color online) Measured polarization $P_{R}$ (diamonds), and total reflectance $R$ (dots), polarization-dependent reflectances $R_{s}$ (squares), and $R_{p}$ (circles) with respective fitted values (continuous curves) of a $\mathrm{MgF}_{2} / \mathrm{SiO}_{2}$ sample at the $\mathrm{H} \mathrm{Ly}-\alpha(121.6 \mathrm{~nm})$ wavelength. calculated reflectivity to the experimental plot (see Table 2 and Fig. 7) are in reasonable agreement with the values determined through the previous $\mathrm{x}$-ray reflectometry, thus providing a useful confirmation.

\section{Conclusion and Outlook}

The method described here allows the determination of polarization-dependent reflectance properties of optical samples in the vacuum-ultraviolet spectral region, in particular shown for (but not restricted to) the 80 to $160 \mathrm{~nm}$ wavelength interval. The extension to the other wavelengths accessible at the particular synchrotron radiation beamline (ranging from 40 to $400 \mathrm{~nm}$ ) only requires the careful and timeconsuming characterization of the beamline polarization dependence on wavelength, which is strongly influenced by the optical configuration (mirror and grating coatings, filters). The method is based on the measurement of the polarization dependence of the responsivity of photodetectors. Since this parameter is generally small, it induces the largest uncertainty contribution, in particular since it is used to transfer polarization properties between two different beamlines. The most critical regime is around $120 \mathrm{~nm}$, where (besides the small value for the detector polarization) the detector responsivity is generally low and effects such as detector inhomogeneity and instability apply. This results in a relatively high overall uncertainty in the measurements.

Further development of polarization-dependent VUV reflectometry at PTB will be significantly promoted by the new possibilities arising in the context of PTB's new electron storage ring for metrological applications of synchrotron radiation, the Metrology Light Source (MLS) [22]. The normal-incidence beamline for reflectometry will be moved to the MLS in 2008 , and will be reconstructed with alignment to the storage ring plane and selectable vertical angular acceptance. Due to the softer synchrotron radiation spectrum at the MLS (compared to BESSY II), it will no longer be necessary to use the off-axis geometry. This will enhance the available photon flux and significantly reduce the uncertainty in reflectance polarization determination. With the help of dedicated multilayer mirrors, such as those presented here, a suitable polarization analyzer for this wavelength region will be developed that will allow to determine the radiation polarization with smaller uncertainties. 
With a polarizer showing a polarization of $\sim 0.8$ at $121.6 \mathrm{~nm}$, the uncertainty contribution from the polarization detector should be reduced to $\sim 2 \%$ (at least comparable to the current value at $160 \mathrm{~nm}$ ). Furthermore, a dedicated VUV undulator beamline will supply monochromatized radiation of high flux, high spectral purity, and a high degree of linear polarization, tunable in the spectral range from UV to EUV. In combination with a suitable instrumentation, not only polarization-dependent reflectance measurements with significantly reduced uncertainties will be possible. Moreover, this will open the field to establish VUV ellipsometry with synchrotron radiation down to the EUV regime.

\section{References}

1. M. Richter, A. Gottwald, F. Scholze, R. Thornagel, and G. Ulm, "Calibration of space instrumentation with synchrotron radiation," Adv. Space Res. 37, 265-272 (2006).

2. M. Richter, J. Hollandt, U. Kroth, W. Paustian, H. Rabus, R. Thornagel, and G. Ulm, "The two normal-incidence monochromator beam lines of PTB at BESSY II," Nucl. Instrum. Methods Phys. Res. A 467-468, 605-608 (2001).

3. J. Schwinger, "On the classical radiation of accelerated electrons," Phys. Rev. 75, 1912-1925 (1949).

4. J. H. Burnett, Z. H. Levine, and E. L. Shirley, "Intrinsic birefringence in calcium fluoride and barium fluoride," Phys. Rev. B 64, 241102R (2001).

5. M. Letz, L. Parthier, A. Gottwald, and M. Richter, "Spatial anisotropy of the exciton level in $\mathrm{CaF}_{2}$ at $11.1 \mathrm{eV}$ and its relation to the weak optical anisotropy at $157 \mathrm{~nm}$," Phys. Rev. B 67, 233101 (2003).

6. R. N. Hamm, R. A. McRae, and E. T. Arakawa, "Polarization studies in the vacuum ultraviolet," J. Opt. Soc. Am. 55, 15601463 (1965).

7. W. R. Hunter, "Design criteria for reflection polarizers and analyzers in the vacuum ultraviolet," Appl. Opt. 17, 12591270 (1978).

8. M. Yang, C. Cobet, and N. Esser, "Tunable thin film polarizer for the vacuum ultraviolet and soft x-ray spectral regions," J. Appl. Phys. 101, 053114 (2007).

9. L. Nahon and C. Alcaraz, "SU5: a calibrated variablepolarization synchrotron radiation beam line in the vacuumultraviolet range," Appl. Opt. 43, 1024-1037 (2004).

10. J. B. Kortright, M. Rice, and K. D. Franck, "Tunable multilayer EUV/soft x-ray polarimeter," Rev. Sci. Instrum. 66, 15671569 (1995).

11. F. Schäfers, H.-C. Mertins, A. Gaupp, W. Gudat, M. Mertin, I.
Packe, F. Schmolla, S. DiFonzo, G. Soullié, W. Jark, R. Walker, X. Le Cann, R. Nyholm, and M. Eriksson, "Soft-x-ray polarimeter with multilayer optics: complete analysis of the polarization state of light," Appl. Opt. 38, 4074-4088 (1999).

12. R. Klein, M. Krumrey, M. Richter, F. Scholze, R. Thornagel, and G. Ulm, "Radiometry with synchrotron radiation at the PTB laboratory at BESSY II," Synchrotron Radiat. News 15, 23-29 (2002).

13. M. Krumrey, M. Kühne, P. Müller, and F. Scholze, "Precision soft x-ray reflectometry of curved multilayer optics," Proc. SPIE 1547, 136-143 (1991).

14. E. I. Ivanov, L. B. Lopatina, V. L. Sukhanov, V. V. Tuchkevich, and N. M. Schmidt, "Silicon $p$ - $n$ junctions with the currentvoltage characteristic of an 'ideal' Shockley diode," Sov. Tech. Phys. Lett. 6, 377-378 (1980).

15. F. Schäfers and M. Krumrey, "REFLEC," Tech. Rep. BESSY 201/96 (Berliner Elektronenspeicherring-Gesellschaft für Synchrotronstrahlung, m.b.h., 1996).

16. A. Gottwald, U. Kroth, M. Letz, H. Schöppe, and M. Richter, "High-accuracy VUV reflectometry at selectable sample temperatures," Proc. SPIE 5538, 157-164 (2004).

17. M.-G. Pelizzo, F. Frassetto, P. Nicolosi, A. Giglia, N. Mahne, and S. Nannarone, "Polarization and higher-order content measurement of a soft-x-ray monochromatized beam with Mo-Si multilayers," Appl. Opt. 45, 1985-1992 (2006).

18. D. Garoli, F. Frassetto, G. Monaco, P. Niccolosi, M. G. Pelizzo, F. Rigato, V. Rigato, A. Giglia, and S. Nannarone, "Reflectance measurements and optical constants in the extreme ultraviolet-vacuum ultraviolet regions for $\mathrm{SiC}$ with different C/Si ratio," Appl. Opt. 45, 5642-5650 (2006).

19. B. Kjornrattanawanich, S. Bajt, and J. F. Seely, "Mo/B4C/Si multilayer-coated photodiode with polarization sensitivity at an extreme-ultraviolet wavelength of $13.5 \mathrm{~nm}$," Appl. Opt. 43, 1082-1090 (2004).

20. F. Bridou, M. Cuniot-Ponsard, and J-M. Desvignes, "Experimental determination of optical constants in the vacuum ultra violet wavelength region between 80 and $140 \mathrm{~nm}$ : a reflectance versus thickness method and its application to ZnSe," Opt. Commun. 271, 353-360 (2007).

21. F. Bridou and B. Pardo, "Automatic characterization of layers stacks from reflectivity measurements. Application to the study of the validity conditions of the grazing X-rays reflectometry," J. Opt. 21, 183-191 (1990).

22. G. Brandt, J. Eden, R. Fliegauf, A. Gottwald, A. Hoehl, R. Klein, R. Müller, M. Richter, F. Scholze, R. Thornagel, G. Ulm, K. Bürkmann, J. Rahn, and G. Wüstefeld, "The metrology light source- the new dedicated electron storage ring of PTB," Nucl. Instrum. Methods Phys. Res. B 258, 445-452 (2007). 